\title{
Si la videovigilancia es la respuesta, ¿cuál era la pregunta? Cámaras, seguridad y políticas urbanas ${ }^{1}$
}

Gemma Galdon-Clavell. Eticas REsearch \& Consulting, Barcelona, España.

RESUMEN | Las cámaras de videovigilancia llevan ańos proliferando en casi todo el mundo. Sin embargo, ni las evaluaciones existentes dan motivos que justifiquen este entusiasmo por las cámaras en la lucha contra la delincuencia, ni las imágenes grabadas han servido de forma determinante en la prevención o resolución de grandes delitos recientes. La pregunta que emerge, pues, es: ¿`cómo es posible que la videovigilancia haya conseguido no solo imponerse sino seguir una trayectoria de expansión, mantener cifras de apoyo popular considerables y generar un consenso favorable inquebrantable entre los responsables políticos, las autoridades policiales y la población en general? A través del repaso a las propuestas académicas realizadas hasta ahora que vinculan la videovigilancia con procesos económicos, sociológicos y políticos más amplios, y con base en los trabajos existentes sobre la evolución de la proliferación de la videovigilancia en España, este artículo pone sobre la mesa, desde una perspectiva sociológica y politológica, la pregunta que nadie parece tener deseos de plantear: ¿por qué la videovigilancia?

PALABRAS CLAVE | política urbana, seguridad ciudadana, innovación tecnológica.

ABSTRACT | Surveillance cameras have been proliferating around the world for decades now. However, evaluations of their benefits and performance, as well as the relatively small impact that they have had on crime prevention lead us to question the generalized enthusiasm for this urban technology. Why have surveillance cameras managed to proliferate and gain widespread acceptance among the population, elected officials and the police when most serious evaluations question their efficacy? By reviewing the literature on closedcircuit television cameras (cctv), this paper links video surveillance to broader economic, sociological and political processes. Studying the proliferation process of surveillance cameras in Spain from a sociological and politological perspective, this paper poses the question no one seems to want to ask: why cctv?

KEY WORDS | urban policy, community safety, technological innovation.

Recibido el 1 de julio de 2013, aprobado el 1 de agosto de 2014

E-mail: gemma.galdon@ub.edu

Correspondencia: C/ Ferlandina 49, 08001, Barcelona, España

1 Este artículo se deriva de la tesis doctoral de la autora (Galdon-Clavell, 2012), realizada con financiación del Comissionat per a Universitats i Recerca, del Departamento de Innovación, Universidades y Empresa (DIUE), de la Generalitat de Catalunya y del Fondo Social Europeo. 


\section{Introducción}

El 11 de marzo de 1995, el New York Times publicó una nota titulada "Cámaras indiscretas capturan sospechosos", que narraba la experiencia de un reportero en una sala de control de videovigilancia de una ciudad británica:

Liverpool, Inglaterra - Aún no habían tocado el 3 cuando la llamada irrumpió en el centro de control, una voz biselada a través del walkie-talkie: cuatro jóvenes caminaban por la zona comercial central, actuando de manera sospechosa. "Ya estamos", dijo el guardia de seguridad, con los ojos en la imagen de los cuatro jóvenes que, con aire arrogante, iban robando manzanas de las tiendas de fruta, sin saber que alguien los seguía con atención (...). La principal preocupación de los guardias (...) era seguir los movimientos de los ladrones, prostitutas y carteristas reincidentes. Durante 20 minutos, las cámaras los siguieron. En varias ocasiones, el grupo se detuvo, como para supervisar el terreno, mientras uno de ellos entraba en una tienda. Los guardias, desde la sala de control, buscaban minuciosamente cualquier rastro de robo, y esa tarde acabaron alertando a la policía. Así que al dar vuelta a la esquina, los jóvenes se encontraron con un policía esperándolos. Este tomó nota de sus nombres y preguntó por radio si alguno de ellos tenía antecedentes. Por lo que captan las cámaras, parece que antes de soltarlos los amonestó. Continuaron su marcha, pero alejándose del centro, lejos de la mirada de las cámaras y con menos humos al caminar².

Es muy común leer y escuchar historias relacionadas con videovigilancia que reproducen el punto de vista de este artículo, donde se describe el funcionamiento de una de las primeras cámaras de vigilancia instaladas en Gran Bretańa con el fin de vigilar un espacio público (en este caso, una zona comercial en el centro). La imagen de la cámara como un perro guardián "neutral" de nuestro entorno no es de ninguna manera excepcional, y pone sobre la mesa toda una serie de preguntas que a menudo permanecen ocultas en los discursos que, como el que acabamos de reproducir, pretenden hacerse eco del "sentido común" y transmitir la idea de que la mirada del ojo electrónico solo debe provocar temor a quien tiene algo que ocultar.

Los protagonistas de la noticia del New York Times, sin embargo, no parecen tener nada que ocultar, más allá de su juventud y arrogancia, que en todo caso no podrían ser consideradas delitos. Sin embargo, que esto sea lo que les hace sospechosos a la vista del controlador de la cámara no aparece cuestionado en el relato. El autor parte, tal vez inconscientemente, de tres presunciones: por un lado, que aquellos que cometen crímenes son fácilmente identificables a través de los ojos de la cámara y que quienes realizan el visionado "ya saben de quiénes se trata"; por otro lado, que "eliminar" a estos elementos de ciertas áreas para que la gente se sienta segura es legítimo y deseable; y, finalmente, que no supone ningún problema el que a ciertas personas se les transmita reiteradamente el mensaje de que su presencia no es bienvenida en ciertas zonas comerciales urbanas.

En el imaginario colectivo (o como mínimo en el mediático), la imagen comercial de la videovigilancia, movilizada por empresas de seguridad y por organismos

Este fragmento, como el resto de citas textuales, es una traducción propia. 
municipales de muchos países, parece haberse impuesto. Se ha extendido el discurso que presenta las cámaras como una herramienta útil para la prevención y reducción de la delincuencia, como una tecnología neutra que reproduce los "ojos sobre la calle" de los que hablaba Jacobs (1961/1992), y que es eficaz sobre todo en la revitalización y control de espacios degradados y/o comerciales.

No es difícil, pues, encontrar descripciones de las bondades del ojo electrónico en estos términos, como ponen en evidencia los siguientes fragmentos de diferentes documentos elaborados por las autoridades de Glasgow (Gran Bretańa) y Madrid (España):

La videovigilancia en los espacios públicos es clave en el programa de seguridad comunitaria de Glasgow, cuyo objetivo es contribuir a la reducción de la delincuencia, la reducción del miedo a la delincuencia y el abordaje de temas comunitarios más amplios, como el vandalismo, los grafitis, los carteles y los incendios provocados. (Glasgow Community \& Safety Services, www.saferglasgow.com)

48 cámaras vigilarán la zona que va de la plaza Tirso de Molina hasta la Glorieta de Embajadores. Medida de carácter disuasivo, incrementará la seguridad, mejorará la calidad de vida de los vecinos (...). "Es una buena noticia -señala [Pedro Calvo, delegado de Seguridad y Movilidad]-. La videovigilancia tendrá un carácter disuasorio tanto para los problemas de convivencia como para los relacionados con la comisión de delitos y faltas. Consecuentemente, tanto los vecinos de la zona, como los comerciantes, como los que la visitamos a menudo, mejoraremos en seguridad y calidad de vida. ("Videovigilancia para Lavapiés". Nota de prensa del Ayuntamiento de Madrid, 26 de mayo de 2009).

Pero no son solo las autoridades locales las que reproducen estos discursos. También los miembros de los cuerpos de seguridad y representantes de instituciones del ámbito estatal a menudo dan por sentado que las cámaras de seguridad multiplican la capacidad de control del espacio urbano (Koskela, 2003, p. 299) y asumen, sin cuestionarlos, los beneficios de la videovigilancia:

Las cámaras de circuito cerrado de televisión atrapan a criminales. Ven los delitos, identifican a los delincuentes y contribuyen a la captura de los culpables. La difusión de esta tecnología significa que más centros urbanos, recintos comerciales, espacios de negocios y zonas de aparcamiento se convertirán en zonas donde los delincuentes no osarán entrar (...) la videovigilancia es un maravilloso complemento tecnológico al trabajo policial (...) un policía de Liverpool afirmaba que su sistema de veinte cámaras era como tener veinte agentes más de servicio 24 horas al día, tomando notas de forma constante. (Michael Howard, secretario de Interior, responsable de la financiación estatal de la videovigilancia. En Norris, 2003, p. 254).

Como enfatizan muchos autores ${ }^{3}$, esta visión, basada en una eficiencia que se presupone y una neutralidad que no se cuestiona, actúa más bien como cortina de humo ante la posibilidad de desarrollar una comprensión más amplia del fenómeno de la vigilancia; una lectura que aborde cuestiones relacionadas con temas de más amplio

Véase la sección siguiente para un listado de autores y trabajos específicos. 
espectro, como el control social, los cambios sociales recientes en la percepción y reacción legal ante el delito y el desorden urbano, el papel de la tecnología y de los intereses económicos que se mueven a su alrededor en las sociedades y la política actuales, la crisis institucional de la modernidad tardía; también las externalidades políticas, sociales y relacionales de la intervención tecnológica en entornos urbanos, la arquitectura de mimetización de políticas en la globalización, el nuevo rol de las ciudades en la construcción del discurso "securitario" global o los realineamientos de poder en un contexto de governance.

El motor del artículo es preguntarse cómo es posible que la videovigilancia haya conseguido no solo imponerse, sino seguir una trayectoria de expansión, mantener cifras de apoyo popular considerables y generar un consenso favorable inquebrantable entre los responsables políticos, las autoridades policiales y la población en general.

Las hipótesis que se plantean son:

- La videovigilancia se impone por ser una medida de aplicación inmediata.

- Se expande por el technological fix (búsqueda de soluciones tecnológicas mágicas) y los discursos acríticos y creyentes en su eficacia, que a su vez derivan en altas tasas de aceptación de esta solución tecnológica por parte de la población.

- Al ser una medida que puede tomarse desde el ámbito local, la videovigilancia permite mostrar capacidad e iniciativa municipal en un campo tradicionalmente monopolizado por las políticas estatales.

En el siguiente apartado se examina desde la vertiente teórica cómo evoluciona el discurso acrítico sobre la videovigilancia hacia una necesidad de la evaluación de la eficacia y la eficiencia. A continuación, en la tercera sección, se retoma el debate sobre la gobernanza y cómo la administración de nivel local es un actor clave en la implementación de la videovigilancia. El estudio de caso de referencia es España, por ser un país mediterráneo (no anglosajón) y postotalitario, con una relación específica con la vigilancia estatal. En la sección sobre la aceptación pública de la videovigilancia en Espańa, los temas relacionados con la aceptabilidad se apoyan empíricamente y con el afán de vislumbrar tendencias a partir de los datos del Centro de Investigaciones Sociológicas. Finalmente se apuntan las conclusiones, en las que se revisan las hipótesis planteadas y se responde, en la medida de lo posible, a la cuestión que da sentido a esta contribución: el porqué de la vigilancia.

\section{La eficiencia y eficacia de la videovigilancia: evaluaciones y datos en perspectiva teórica}

El discurso oficial basado en las expectativas y los presupuestos impide la articulación de un debate que incorpore el creciente corpus académico (Bétin, Martinais \& Renard, 2003; Cameron, Kolodinski, May \& Williams, 2008; Deisman, 2003; DíezRipollès \& Cerezo, 2009; Ditton, 2002; Douillet, Dumoulin \& Germain, 2009; Gill \& Spriggs, 2005; Groombridge, 2008; Helimann, 2003; Keval \& Sasse, 2008; Murakami Wood, Ball, Lyon, Norris \& Raab, 2006; Webster, 2009; Williamson \& McAfferty, 2000), literatura que pone en duda la utilidad de los circuitos cerrados 
de televisión, tanto en la disuasión de la delincuencia como en la mejora de la intervención policial y la percepción social de los niveles de seguridad. Los trabajos provenientes de este ángulo de estudio evaluativo de las políticas de seguridad, pese a que algunos de ellos ya son bastante antiguos (Grandmaison \& Tremblay, 1995; Webb \& Laycock, 1992), han dejado de ser residuales para conseguir, recientemente, un impacto significativo tanto en los medios de comunicación como en el discurso oficial de ciertos actores implicados. No obstante, la contundencia de los datos parece no haber alterado la apuesta por la proliferación de cámaras.

Frente a esta tendencia generalizada a dar por hecho el impacto beneficioso de las cámaras, la evaluación emerge como una medida necesaria y urgente. En el ámbito, sobre todo, de las políticas públicas, la toma de decisiones debería depender de la disponibilidad de un capital significativo de información válida y contrastada sobre los efectos potenciales de cualquier iniciativa, instrumento o programa. Que este escenario ideal de deliberación, racionalidad perfecta y secuencialidad coincide poco con la realidad de los procesos de decisión política es una obviedad (John, 1998; McCann, 2010; Pressman \& Wildavsky, 1984). En el caso de la videovigilancia, ante la ausencia de evidencias científicas que permitan reforzar el entusiasmo hacia la capacidad de la videovigilancia para intervenir de forma decisiva en los patrones criminales o sobre el sentimiento de inseguridad, los debates sobre estos dispositivos han tendido a basarse en expectativas, inferencias y conjeturas (Deisman, 2003; Gill, Bryan \& Allen, 2007). En 1994, por ejemplo, cuando Groombridge y Murji publicaron uno de los primeros trabajos que planteaba dudas sobre el éxito de los circuitos cerrados de televisión en Gran Bretańa, mencionaron que a pesar de los datos, la videovigilancia parecía haber adquirido estatus de "dogma de fe", y mostraban sorpresa ante la falta de voluntad de plantear abiertamente si las cámaras funcionaban, si justificaban su coste o si tenían efectos perversos.

Este escenario extremo, que dominó en la relación entre el discurso sobre la seguridad ciudadana y la videovigilancia en los ańos noventa, empieza a ser superado, sobre todo en el mundo anglosajón (Hempel \& Töpfer, 2004; Murakami Wood, 2009; Webster, Töpfer, Klauser \& Raab, 2012). Aunque se siguen señalando deficiencias metodológicas (Armitage, 2002), en los últimos lustros los estudios independientes que cuestionan el impacto de la videovigilancia sobre la delincuencia y la sensación de inseguridad son cada vez menos anecdóticos, encargados incluso por las mismas instituciones que promueven su adopción, como el Home Office (Gill \& Spriggs, 2005; Tilley, 1993; Welsh \& Farringdon, 2002) y el Comisionado para la Información británicos (Murakami Wood et al., 2006), el Departamento de Justicia de Estados Unidos (Ratcliffe, 2006) o la policía canadiense (Deisman, 2003). Diversos autores y estudios han puesto sobre la mesa no solo la importancia de evaluar la eficacia, eficiencia y economía de las cámaras, sino también la necesidad de revisar algunas de las evaluaciones elaboradas a corto plazo o de manera poco rigurosa (Fyfe \& Bannister, 1996), contextualizar los motivos y las interacciones que pueden contribuir a explicar el éxito o el fracaso de la videovigilancia (Tilley, 1993; Groombridge, 2008) y poner en cuestión los efectos a largo plazo de las cámaras en el espacio público, incluso en aquellos casos en que los primeros estudios revelen una disminución inmediata de los incidentes (Armitage, 2002; Gill \& Spriggs, 2005; Welsh \& Farringdon, 2002). 
$\mathrm{Al}$ igual que en el ámbito anglosajón, donde hace tiempo que se elaboran evaluaciones, desde algunos años contamos con trabajos de análisis comparativo entre resultados de varios estudios, es decir, evaluaciones de evaluaciones. Existen más de veinte en el caso de Phillips (1999), trece en Gill y Spriggs (2005), dieciocho en Welsh y Farringdon (2002) o cuarenta y cuatro en Cameron et al. (2008), por citar algunos ejemplos.

En general, lo que constatan estas evaluaciones internacionales es que los sistemas de videovigilancia en el espacio público pueden contribuir a la reducción de la delincuencia en dicho ámbito, pero de manera desigual: más en lugares de poca actividad social y poco control informal, como los aparcamientos aislados, por ejemplo, que en centros urbanos y zonas residenciales, sobre todo en el caso de los Estados Unidos (Short \& Ditton, 1995; Tilley, 1993). El impacto de las cámaras, además, parece ser mayor cuando hay carteles indicativos que pueden generar un efecto disuasorio y cuando la zona vigilada es un espacio de acceso limitado (Díez-Ripollés \& Cerezo, 2009). Los estudios indican también que el impacto de la videovigilancia es desigual, y que ciertas categorías delictivas se ven inalteradas por la presencia de las cámaras, como los delitos violentos y la delincuencia contra las personas (Hier, 2010, p. 222), si bien en el caso de los delitos contra la propiedad los datos son algo más alentadores. Además, como seńala Deisman (2003), por cada estudio que demuestra algo, existen otros similares que establecen lo contrario o cuestionan los resultados previos, ya que las bajas tasas de impacto de la videovigilancia sobre la delincuencia o la sensación de inseguridad, incluso en los casos en que sí se constata una reducción, hacen difícil llegar a resultados concluyentes que permitan descartar el impacto de factores contextuales u otras medidas securitarias sobre los incidentes y su percepción. Las cifras que se mencionan, que suelen rondar un $4 \%$ de disminución de los ilícitos en las zonas videovigiladas, son demasiado bajas para concluir que la reducción sea consecuencia directa de la instalación de cámaras (Welsh \& Farringdon, 2002).

La evidencia científica sobre la relación entre delincuencia y criminalidad, pues, lo que deja claro es que los resultados son mixtos, poco claros y, a veces, directamente divergentes, y que en muchos casos los efectos positivos y las externalidades negativas se contrarrestan mutuamente (Welsh \& Farringdon, 2003). Lo que sí parece generar consenso, sin embargo, es que las cámaras no tienen el mismo impacto sobre todas las categorías delictivas ni en todas partes, de modo que, como solución generalizada a problemas de seguridad ciudadana, la videovigilancia es manifiestamente deficiente (Cameron et al., 2008; Ratcliffe, 2006) y puede generar una serie de externalidades negativas que hay que tener en cuenta (Dixon, Levine \& McAuley, 2003; Mazerolle, Hurley \& Chamlin, 2002; Von Hirsch, 2000).

De manera más específica, en el contexto británico los estudios sugieren que el impacto de la videovigilancia sobre los delitos es modesto, y que en muchos casos es evidente que la presencia de las cámaras produce un efecto desplazamiento de la delincuencia a zonas cercanas (Ditton, 2002; Gill \& Spriggs, 2005). Los resultados son muy similares en el ámbito francés, donde la literatura científica sobre la videovigilancia parece estar en un momento de auge y aporta un número significativo tanto de estudios de caso como de evaluaciones (Bétin et al., 2003; Douillet et 
al., 2009; Heilmann, 2003; Levan, 2009) ${ }^{4}$. En el caso de Estados Unidos, algunos estudios constatan que la iluminación de las calles es más eficaz en la reducción del vandalismo y los robos que las cámaras, y que los resultados en relación con el efecto desplazamiento son contradictorios (Welsh \& Farringdon, 2003). Groombridge (2008), por su parte, concluye que, de nuevo en el contexto británico, la videovigilancia de espacios públicos no justifica la inversión que exige, y Webster (2009) alerta respecto de que la debilidad de los datos que justifican la efectividad de los circuitos cerrados de televisión está poniendo en cuestión el desarrollo futuro de esta solución urbana.

Deisman (2003), además, profundiza en el cuestionamiento de uno de los elementos que, junto con la eficiencia de recursos y la detección a posteriori (que abordaremos seguidamente), más recorrido ha tenido en la justificación de la videovigilancia como herramienta de lucha contra la delincuencia: su potencial efecto disuasorio, que está vinculado tanto con la supuesta capacidad de las cámaras para alterar el comportamiento de la ciudadanía, en el sentido disciplinario que teoriza Foucault (1975/1995), como, desde una perspectiva más criminológica, con el efecto desincentivador sobre los posibles delincuentes que supuestamente realizan un cálculo de coste/beneficio antes de actuar. En el discurso político y mediático sobre la videovigilancia, la falta de evidencias científicas sobre la utilidad de las cámaras a menudo se salva apelando a este abstracto efecto disuasorio, difícil de cuantificar. La disuasión, además, se basa en una serie de expectativas sobre el comportamiento, los procesos cognitivos y las motivaciones, que hay que poner sobre la mesa.

Para afirmar que las cámaras tienen efectos disuasorios, es necesario asumir toda la cadena de expectativas que hacen posible este efecto: que el potencial perpetrador es consciente de la existencia de cámaras en un espacio concreto; que el individuo dispuesto a cometer un delito considera que la presencia de estas cámaras reduce sus posibilidades de éxito o de impunidad, o asume que las cámaras están monitorizadas y, por lo tanto, será identificado y detenido inmediatamente; que el acto delictivo que se quiere cometer es fruto de un cálculo premeditado; que el potencial perpetrador decide, como consecuencia de este cálculo, desistir del acto delictivo y, por último, que es capaz de controlarse, poner la razón por delante del impulso y, por lo tanto, desistir. Perspectivas de este tipo, relacionadas con las teorías de la elección racional y de la oportunidad que se han impuesto en los últimos treinta años (Garland, 2001), han tenido un impacto significativo en las políticas de seguridad y en el discurso que envuelve la elección de la videovigilancia como herramienta política de reducción de la delincuencia. Su ajuste a la realidad, sin embargo, es cuestionable (Cohen, March \& Olsen, 1972).

Antes de abordar otros aspectos vinculados con la eficacia y eficiencia de las cámaras, podemos hacer una comparación inicial de los resultados y conclusiones que acabamos de mencionar con la situación en España. Si bien el desinterés por parte de las autoridades públicas para llevar a cabo evaluaciones serias del impacto de la videovigilancia en el contexto español es manifiesto, en 2007 la Universidad

Para una visión general de la contribución francesa a la teorización de la vigilancia, véase Klauser (2009). 
de Málaga llevó a cabo una medición de la eficacia de las cámaras en la reducción de la delincuencia en la ciudad. Realizado de manera independiente y a partir de una metodología escrupulosa y cuasi- experimental (Miller, 2006), este estudio nos permite aterrizar el debate sobre las cámaras en nuestro entorno concreto, a partir del seguimiento del proceso previo y posterior a la instalación de diecisiete cámaras en el centro de la capital andaluza, a petición de los comerciantes de la zona y con el apoyo y la financiación del Ayuntamiento.

Las conclusiones de Cerezo y Díez-Ripollès (2010) son significativas. En cuanto a las estadísticas policiales, observan un menor incremento de los actos delictivos en la zona vigilada, pero prácticamente imperceptible $(1,9 \%)$, combinado con un aumento del $14,6 \%$ en las zonas cercanas a las calles videovigiladas, lo que evidencia un marcado efecto desplazamiento (ibíd., p. 4). En cuanto a la tipología de delitos, el efecto no es visible: la distribución y el peso específico de cada categoría delictiva se mantienen inalterados antes y después de la instalación de las cámaras (ibíd., p. 5).

En relación con la victimización ${ }^{5}$, en el trabajo de Díez-Ripollés y Cerezo (2009) los resultados son similares en cuanto a los datos totales de delitos declarados, y constatan un menor aumento en la zona vigilada $(0,8 \%)$ que en la zona de control $(2,8 \%)$. Por categorías, el estudio determina que, en el caso de robos a personas (primera categoría en importancia), las encuestas revelan una diferencia significativa: aumentan un $12,5 \%$ en la zona no vigilada, y solo un $5,4 \%$ en la zona de tratamiento. A pesar de no apreciarse reducción, pues, la diferencia empieza a ser significativa.

En cuanto a la percepción de inseguridad, los autores registran pocos cambios tras la instalación de las cámaras, y concluyen que existen "serias dudas sobre la utilidad [de la videovigilancia] para conseguir los objetivos que se pretenden” (DíezRipollés \& Cerezo, 2009, p. 25).

El abismo entre las expectativas y el impacto real de la videovigilancia ha ido tomando forma, precisamente, entre los que viven más de cerca su operativa: los policías. En 2008, por ejemplo, el responsable de la National cctv Strategy británica, el agente Graeme Gerrard, declaraba, en relación con las expectativas depositadas en la videovigilancia:

La mayor parte de la presión [para instalar cámaras] proviene de la población (...). Es posible que terminen decepcionados (...) no detiene la mayor parte de los delitos. Creo que es posible que se les haya inducido a error en términos de la cantidad de delitos que la videovigilancia puede prevenir. (Telegraph, 19 de enero de 2008)

Pocos meses después, la misma publicación reproducía las declaraciones de uno de los expertos en circuitos cerrados de televisión de Scotland Yard, que afirmaba que la videovigilancia era un "fiasco total", ya que solo un 3\% de los robos en la calle se había podido resolver identificando el perpetrador gracias a las imágenes recogidas por las cámaras (Hempel \& Töpfer, 2009, p. 158). Más recientemente, en 2009, la Policía Metropolitana londinense hacía público que solo una de cada mil cámaras

Las encuestas de victimización permiten recoger datos sobre delitos a partir de las declaraciones de las personas y no solo de las denuncias presentadas. 
contribuye a la resolución de casos delictivos, a la vez que ponía precio a cada una de estas intervenciones: 20.000 libras esterlinas ${ }^{6}$.

Si tenemos en cuenta, como plantean Hempel y Töpfer (2009), que se calcula que el Ministerio de Interior británico ha gastado cerca de 500 millones de libras en la infraestructura vinculada a la videovigilancia (lo que supone más de tres cuartas partes del total del presupuesto para la prevención de la delincuencia), y que, en muchos casos, las instalaciones no cumplen con la normativa de respeto a derechos fundamentales, como la intimidad y la propia imagen ${ }^{7}$, el sacrificio de libertades y garantías democráticas en aras de la seguridad en que a menudo se basa la justificación del aumento de medidas de seguridad en la vida cotidiana parece dejar de tener sentido: la renuncia a la libertad no se ve compensada ni recompensada por una mayor seguridad.

La pregunta que emerge, pues, es: ¡cómo es posible que la videovigilancia haya conseguido no solo imponerse, sino seguir una trayectoria de expansión, mantener cifras de apoyo popular considerables y generar un consenso favorable inquebrantable entre los responsables políticos, las autoridades policiales y la población en general?

Si las evidencias fueran contradictorias, o las externalidades marginales, el estudio de la videovigilancia como instrumento de las políticas públicas de seguridad podría reducirse a la valoración de la relación entre costes y beneficios, o entre expectativas y resultados. La emergencia de un sólido corpus científico que cuestiona el relato construido alrededor de la necesidad de instalar cámaras para combatir la delincuencia obliga a ir más lejos: a dejar de ver la videovigilancia como una respuesta a la inseguridad para convertirla en una pregunta; a buscar los ejes que articulan y dan sentido a la economía política de la videovigilancia; a poner sobre la mesa la pregunta que nadie parece tener deseo de plantear: ¿por qué la videovigilancia?

\section{La proliferación de la videovigilancia: drivers y elementos contextuales}

Otras publicaciones se han centrado en la descripción del proceso concreto de adopción y proliferación de la videovigilancia en España (Galdon-Clavell, 2011; Galdon-Clavell, Zuloaga \& Romero, 2012). En esta ocasión, el objetivo es centrarse en algunos de los elementos contextuales que pueden contribuir a la comprensión del porqué de la adopción y proliferación de una política que parece incapaz de cumplir las expectativas que en ella se depositan. Con el fin de explorar estos elementos ligados a lo que podríamos llamar la "economía política" de la videovigilancia, se abordan aspectos vinculados al papel de los municipios y ciudades (en oposición al protagonismo regional o estatal) en la apuesta por la videovigilancia; al rol que pueden desempeñar los procesos de transferencia de políticas (policy transfer) en el reforzamiento de esta apuesta; $y$, finalmente, en el papel que juegan la redefinición de los miedos urbanos y el progresivo divorcio entre la inseguridad objetiva y el sentimiento de inseguridad. Para ello se toma el caso español.

“1,000 cCTV cameras to stop just one crime, Met Police admits", Telegraph, 25 de agosto de 2009. 
Es evidente el creciente protagonismo de los representantes políticos locales en la definición de la agenda política supralocal (Sassen, 2006), incluida la securitaria (Mitchell \& Beckett, 2008; Raco, 2003). El caso español, donde la apuesta por la videovigilancia es siempre municipal, confirma que dicha opción puede adquirir relevancia regional y estatal -a pesar de tomar forma como política de amplio espectro- a través de la suma de iniciativas locales poco conectadas entre sí, con una capacidad de transferencia de experiencias limitada y sin ninguna bolsa de financiación compartida (Galdon-Clavell, 2012).

Aunque algunas de las características que emergen en el proceso de proliferación de las cámaras de seguridad en España son específicas del caso español ${ }^{8}$, este "nuevo localismo" (Goetz \& Clarke, 1993; Le Galès, 1999) entronca claramente con tendencias más generales. La ciudad, por tanto, emerge como el espacio donde toma forma el paso de la producción a la financiarización, de la reproducción social a la privatización (Brenner \& Theodore, 2002; Sassen, 1991).

En los estudios sobre la vigilancia, los autores han tendido a ver la esfera local como el lugar de donde emerge un nuevo 'liderazgo neoliberal' (Coleman, 2004; Greenberg, 2008) y a considerar los circuitos cerrados de televisión como un elemento primordial en la estrategia general de domesticación y control del espacio público que ha caracterizado las políticas urbanas occidentales durante los últimos treinta años (Fyfe, 1998). En muchos casos, pues, el ámbito local aparece tratado como el campo de batalla de tensiones supralocales, el espacio donde las presiones globales toman la forma de partenariados público-privados e imponen una redefinición de las políticas urbanas en términos economicistas, priorizando una seguridad excluyente y reivindicando la gestión del desorden a través de la vigilancia y el control, por encima del abordaje de las cuestiones sociales (Cochrane, 2007).

El caso de la videovigilancia en Espańa confirma sin lugar a dudas la existencia de un proceso de descentralización y de aumento de la autonomía del ámbito local (Blanco, 2004; Jones \& Stewart, 1985; Solesbury, 1993). Esto, sin embargo, no es consecuencia principalmente de la cesión o devolución a los entes locales de competencias sobre seguridad tradicionalmente reservadas al Estado (muchos municipios españoles cuentan desde hace años con fuerzas policiales propias y concejalías de seguridad), sino de la apropiación activa por parte de estos de un papel de liderazgo en la propuesta de la videovigilancia y su adopción como solución política a problemas de seguridad. En el caso de Cataluña, por ejemplo, ninguno de los municipios que solicitó videovigilancia entre 2000 y 2012 ha recibido financiación autonómica o estatal para la instalación de cámaras en el espacio público, y los empresarios, políticos y partidarios de la videovigilancia no se han beneficiado de la existencia de un discurso legitimador de esta solución a nivel supralocal, tal como ha ocurrido, por ejemplo, en Francia y Gran Bretańa (Fussey, 2004; Rochette \& Marchandet, 1998). En realidad, la situación es la contraria: la videovigilancia como herramienta de lucha contra el incivismo y la pequeña delincuencia es un tema que se ha construido en el nivel local, a partir de la apropiación por parte

Para un análisis en profundidad de este caso, véase Galdon-Clavell (2012), y Galdon-Clavell y Pybus (2011). 
de poderosos actores públicos y privados locales de la videovigilancia como herramienta de intervención y como discurso político.

En este proceso es relevante el hecho de que el discurso justificador de la videovigilancia sea cambiante, se adapte a las circunstancias de cada momento y priorice la consecución de resultados (la instalación de cámaras) por encima de la coherencia y la consistencia argumental. Así, en el caso español, las cámaras han ido perdiendo con el tiempo su vinculación inicial con el discurso antiterrorista. De todos los casos analizados en Galdon-Clavell et al. (2012), la referencia a la amenaza terrorista solo aparece en dos (Granada y Barcelona), y siempre de forma puntual y poco relacionada con eventos significativos. En la gran mayoría, la necesidad de cámaras se justifica a partir de la existencia de conflicto, incivismo, vandalismo, tráfico de drogas a pequeña escala, consumo de alcohol, ruido, etcétera. Este hecho subraya el proceso que describíamos, por el cual las ciudades dejan de limitarse a ser espacios donde los procesos globales encuentran eco o las políticas estatales son ejecutadas para exigir el desempeño de un rol activo, haciendo presión con el fin de insertar soluciones políticas en la agenda supralocal.

En el contexto de las políticas de seguridad, esta capacidad de las autoridades municipales para convertirse en portavoces de nuevas iniciativas y dejar atrás un papel basado en la subordinación a las agendas políticas estatales, sin embargo, no es ni nueva ni exclusiva de nuestro entorno. Irrumpió claramente en la escena internacional durante la gestión de Rudolph Giuliani en la ciudad de Nueva York. Giuliani se convirtió, en 1993, en el primer alcalde estrella gracias a una campańa centrada en el discurso securitario y la apuesta por la mano dura. Su propuesta, basada en la aplicación de los principios de la teoría de las ventanas rotas (Kelling \& Coles, 1996; Wilson \& Kelling, 1982) y la idea de que la policía debe intervenir no solo de forma reactiva ante la comisión de un ilícito, sino también durante las fases "previas" vinculadas al desorden urbano y los comportamientos molestos, consiguió entrar en la agenda securitaria global (Cochrane, 2007; Dixon \& Maher, 2005), precisamente por el hecho de articularse en un momento en que las ciudades estaban empezando a conseguir un protagonismo renovado, sobre todo económico, en el nuevo escenario internacional (Fox Gotham, 2002; Lefevbre, 1996; Zukin, 1995).

Es innegable que las ciudades se sitúan hoy en la vanguardia de muchos cambios políticos. En el caso de las políticas de seguridad y control urbano, son a menudo los municipios los que lideran la definición del campo de actuación y las alternativas políticas posibles, como demuestra claramente el impacto de la propuesta de Rudolph Giuliani. De hecho, es posible que la capacidad de liderazgo y contagio, de articulación de redes propias de intercambio de experiencias y de aprendizaje de políticas (policy learning) que se articula alrededor de las políticas de seguridad, sea uno de los temas que más ha contribuido a la consolidación del espacio de las grandes urbes en el escenario de la gobernanza global.

En definitiva, el caso español muestra claramente que la videovigilancia puede emerger como alternativa política incluso en ausencia de complicidad con instancias supralocales. Aunque en los casos estudiados hasta ahora en la literatura internacional el papel del ámbito urbano, como protagonista si no de la iniciativa sí del 
filtrado concreto de la apuesta por las cámaras, ha tendido a ignorarse, la indiscutible emergencia de la ciudad como un espacio de relativa autonomía y con capacidad de liderazgo político pone sobre la mesa la necesidad de estudiar su papel no solo como espacio físico de recepción de políticas, sino también como socio necesario y, a menudo, activo animador del desarrollo de políticas de control del espacio público.

Finalmente, al atractivo de este tipo de soluciones políticas rápidas y fácilmente legitimables hay que añadir el elemento de fascinación que provoca la tecnología, y el creciente recurso a las soluciones tecnológicas (technological fix) ante problemas sociales. La fe en las propiedades de la tecnología, y la utilidad de los artefactos técnicos para ser mostrados como evidencia "física" de la acción política, abren paso a la consolidación de un determinismo tecnológico que justifica la ausencia de evaluación de resultados y la falta de rigor y proceso en la toma de decisiones (Galdon-Clavell \& Pybus, 2011). El potencial simbólico de las cámaras para transmitir una imagen de capacidad y acción política emerge como uno de los motores principales, aunque inconfesado, de la promoción de la videovigilancia en los espacios públicos.

\section{La aceptación pública del ojo electrónico en España}

A pesar de la proliferación de cámaras en espacios públicos y semipúblicos, la videovigilancia es un tema que no ha sido objeto de debate ni en Espańa ni en otros países fuera del ámbito anglosajón. No lo ha sido ni en los medios de comunicación, ni en los espacios de representación política ni en la academia. El vernáculo oficial creado alrededor de la videovigilancia, falto de datos y exigencias de rigor, tiende a reproducir narrativas basadas en expectativas y lugares comunes más que en evaluaciones independientes de impacto y resultados. Cabe esperar, por tanto, que la percepción pública sobre las cámaras refleje necesariamente la inexistencia de narrativas alternativas.

Los únicos datos disponibles hasta el momento de redacción de este artículo son tres oleadas del Centro de Investigaciones Sociológicas, España (cis, 2008, 2009 y 2011). En general, las encuestas muestran un elevado nivel de apoyo al uso de cámaras, con cifras similares a las extraídas de encuestas de otros países (Leman-Langois, 2009).

Según los datos de 2009 (los más completos), del 68,7\% de la población española que da apoyo a la videovigilancia, un $66,4 \%$ de los encuestados lo hace porque las cámaras les dan seguridad, el $18 \%$ porque considera que permiten la identificación de delincuentes, y el 15,2\% porque cree que es una forma de prevención de la delincuencia. No obstante, un 10\% rechaza las cámaras, básicamente por la pérdida de privacidad (79,4\%). En cuanto a la localización de las cámaras, no todas las ubicaciones cuentan con el mismo grado de aceptación y oposición: los bancos, los comercios, las guarderías, colegios y hospitales reciben el apoyo más elevado; por el contrario, en el caso de los lugares de trabajo, bares y restaurantes, los espacios de ocio, los espacios públicos y los edificios residenciales, el apoyo es mucho menor (Cuadro 1).

Un hecho relevante y que contradice cierta tendencia catastrofista (o excesivamente optimista, según se mire) es que en Espańa se augura un cambio de tendencia: entre febrero de 2008 y mayo de 2011, el apoyo de la población a la videovigilancia 
ha disminuido prácticamente un 5\% (de 73,2\% a 68,3\%). A su vez, las personas que se sitúan en contra o muy en contra de la videovigilancia pasaron a representar el $13,7 \%$ de la población (eran el 9,5\% en 2008 y 10\% en 2009). Los datos de la encuesta de 2009, que exploran el imaginario que envuelve la videovigilancia, indican que el porcentaje de personas que asocian los circuitos cerrados de televisión con una mayor seguridad y protección y menos miedo ha disminuido de $71,1 \%$ a $66,4 \%$-una reducción similar, por otra parte, al aumento registrado entre aquellos que valoran las cámaras por su capacidad de prevenir delitos (del 11,6\% al 15,2\%).

CUADro I | Respuesta a la pregunta “¿Podría decirme si le parece muy bien, bien, mal o muy mal que se pongan cámaras de seguridad en...?”

\begin{tabular}{|l|c|c|c|c|c|}
\cline { 2 - 6 } \multicolumn{1}{c|}{} & $\begin{array}{c}\text { BIEN O MUY } \\
\text { BIEN (\%) }\end{array}$ & $\begin{array}{c}\text { NI BIEN NI } \\
\text { MAL (\%) }\end{array}$ & $\begin{array}{c}\text { MAL O MUY } \\
\text { MAL (\%) }\end{array}$ & NS/NC (\%) & (N) \\
\hline Bancos & 95,5 & 1,9 & 1,2 & 1,3 & 2.475 \\
\hline Comercios & 88,3 & 5,9 & 3,7 & 2 & 2.475 \\
\hline $\begin{array}{l}\text { Comunidades de vecinos o } \\
\text { urbanizaciones }\end{array}$ & 51,1 & 14,9 & 28,7 & 5,3 & 2.475 \\
\hline Lugares de trabajo & 44,7 & 13,6 & 36,7 & 5 & 2.475 \\
\hline Guarderías y colegios & 77,2 & 8,2 & 11,2 & 3,3 & 2.475 \\
\hline Transporte público & 74,5 & 8,8 & 13,3 & 3,3 & 2.475 \\
\hline Calles (vía pública) & 60,6 & 12,5 & 23,5 & 3,4 & 2.475 \\
\hline Hospitales & 75,1 & 8,5 & 13,2 & 3,3 & 2.475 \\
\hline Bares y restaurantes & 46,2 & 13,3 & 36 & 4,5 & 2.475 \\
\hline Lugares de ocio & 46,2 & 13,3 & 36 & 4,5 & 2.475 \\
\hline
\end{tabular}

FUENTE ELABORACIÓN PROPIA A PARTIR DE DATOS DE CIS (2009)

Es importante destacar que el $30 \%$ de las personas que no muestran un apoyo inequívoco a las cámaras (y un 13,7\% que las rechaza directamente) parece mostrar un activismo creciente, tanto en la denuncia de dispositivos como en la organización de acciones directas. La propia Agencia Española de Protección de Datos (AEPD) concluía en su informe anual de 2009 que "la percepción positiva sobre la videovigilancia va acompañada de una exigencia de garantías para la privacidad" (AEPD, 2009, p. 34). En cuanto a la acción directa para dificultar la extensión de cámaras, se han dado múltiples historias de vandalismo e incluso movilizaciones contra las cámaras, y actos más o menos organizados de sabotaje en Madrid, Santiago de Compostela y Bilbao, entre otras ciudades (Galdon-Clavell, 2012).

Aunque la proliferación de cámaras parece debilitar el marco legal, no se puede afirmar que la normalización de la videovigilancia en calles y plazas esté fomentando una mayor aceptación de dispositivos, sino al contrario. Aunque las cifras no son abrumadoras, en España la generalización del ojo electrónico va acompañada de un sostenido aumento del rechazo y la resistencia, en lugar de la aceptación de su inevitabilidad. La identificación de esta tendencia permite abrir nuevos horizontes en la comprensión de la dialéctica entre sociedad, tecnología y seguridad, más allá 
de determinismos sociales y/o tecnológicos y con la exigencia de un análisis mucho más complejo y profundo de los factores que articulan esta relación.

\section{Conclusiones.}

\section{La cámara de seguridad: ¿̨in en sí misma o estrategia más amplia?}

Si aceptamos que el protagonismo local en las políticas urbanas forma parte de un proceso mayor de reestructuración de la gobernanza, abrimos también la puerta a la conceptualización del ojo electrónico en dinámicas políticas más amplias relacionadas con la gestión del espacio público, la delincuencia y el desorden urbano. Mientras que en la mayoría de países de nuestro entorno la videovigilancia ha sido teorizada y analizada como una política en sí misma (Coleman, 2004; Hier, 2010; sCAN/The Surveillance Studies Centre, 2009), o como la parte más visible de la apuesta por el control social a través de las nuevas tecnologías (Murakami Wood et al., 2006), orientada a proporcionar seguridad en un mundo de riesgos globales, en el caso espańol el discurso de la videovigilancia aparece enmarcado de manera inequívoca en una problemática más amplia y cotidiana: la inseguridad y el incivismo. Esto explica que los lugares privilegiados para la instalación de cámaras en el país sean en muchos casos espacios de ocio juvenil, y que el incivismo aparezca de manera creciente entre los motivos justificadores de la necesidad de videovigilancia tanto en las solicitudes de nuevas instalaciones como en las renovaciones anuales (Galdon-Clavell, 2011). Adicionalmente, las imágenes captadas por cámaras de seguridad más difundidas por los medios españoles son en general escenas de agresiones y malos comportamientos, a diferencia de otros países, donde, en el imaginario público, la cámara se asocia mucho más con la comisión de delitos graves o de actos terroristas.

Asimismo, en el caso español estos temas se relacionan, en el discurso público y mediático, con la inmigración, corroborando así la existencia de una relación entre la sensación de inseguridad (entendida como una variable significativa en la probabilidad de apostar por la videovigilancia) y los porcentajes de población extranjera en los municipios que solicitan cámaras? ${ }^{9}$ La proliferación de los circuitos cerrados de televisión, pues, en el caso espańol aparece vinculada con un aumento del miedo, que es reflejo de una creciente inquietud ante la composición multiétnica de la sociedad, y que a la vez se manifiesta en la demanda de una mayor protección frente a la delincuencia (Rodríguez Borges \& Campos, 2010). El protagonismo local en la generalización de la videovigilancia, entonces, puede ser mejor interpretado en el contexto de otras políticas desplegadas también desde los ámbitos municipales, relacionadas con esta voluntad de abordar la creciente inquietud urbana a partir de políticas orientadas a mostrar mano dura contra la inmigración, a la que se responsabiliza de la inseguridad. Con base en este análisis, la demanda de excluir a las personas indocumentadas del acceso a los servicios públicos, las propuestas de instauración de "certificados de civismo" para la renovación de permisos de trabajo y residencia, o la prohibición de acceder a ciertos espacios con burka, todo ello a

Los datos concretos en los que se sustentan estas afirmaciones pueden encontrarse en GaldonClavell (2012). 
partir de un argumentario centrado en la inseguridad, serían elementos del mismo discurso que justifica la necesidad de videovigilancia y donde se articula un continuum de demandas securitarias interrelacionadas a nivel local.

Otro elemento significativo que pone de relevancia el caso espańol es el hecho de que las iniciativas municipales señaladas han sido a menudo desautorizadas desde instancias políticas supralocales, que han insistido en la necesidad de respetar el marco legal y cumplir el precepto constitucional de no discriminación. Así, la importancia de la videovigilancia per se, como programa y apuesta pública de amplio espectro, capaz de absorber cantidades muy significativas de recursos y guiar políticas, que observamos en países como Gran Bretańa o Francia, no se reproduce en España. En nuestro entorno, las cámaras emergen como un elemento de refuerzo de iniciativas políticas locales vinculadas a la voluntad de controlar el espacio público, pero sin llegar a tener entidad ni protagonismo suficientes como para convertirse en una política en sí mismas. Pruebas de ello son la poca e irregular presencia del fenómeno en el debate sobre el espacio urbano y la seguridad, la escasa prioridad del tema en la agenda política y policial y las cifras totales de dispositivos públicos de vigilancia instalados en España, que es probable que no superen los 5.000 (excluyendo cámaras perimetrales y de transporte público). En nuestro entorno inmediato, pues, la videovigilancia desempeńa un papel de complemento o parte integrante de una agenda securitaria local más amplia, que mezcla preocupaciones vinculadas al uso intensivo del espacio público por parte de los jóvenes, a la creciente presencia pública de los "consumidores fallidos" (Bauman, 2000) y a la cristalización de las angustias contemporáneas en la figura del inmigrante.

Por lo tanto, a la comprensión del porqué de la videovigilancia y a la descripción del creciente rol que ejercen los entes locales en la definición de la agenda política en general, y de la securitaria en particular, hay que sumar una complementariedad de la videovigilancia con otras políticas de control del espacio público, vinculadas al aumento de la percepción de la inseguridad y la erosión de la tolerancia y el respeto mutuo (Bannister \& Kearns, 2009; Millie, 2009), que no aparece descrita de manera tan directa ni explícita en otros casos recogidos en la literatura internacional.

La aceptabilidad de la instalación de cámaras de videovigilancia en espacios públicos y semipúblicos no ha sido objeto de debate España. Como resultado, el no cuestionamiento es la tónica general. Examinando los datos del cis, se constata que la población acepta mayoritariamente la instalación de dispositivos, aunque bien es cierto que las percepciones sobre su adecuación y justificación cambian en función de los espacios y las actividades que se desarrollan en ellos (siendo los bancos y los comercios los que recogen un mayor consenso, y los espacios de ocio, los que menos). De forma expresa, la presencia de cámaras incrementa la percepción de seguridad y protección, a la vez que las cámaras se consideran una buena medida de prevención contra la delincuencia. La oposición aglutina a un tercio de la población, cuyo rechazo se vincula en gran medida a la conciencia de pérdida de privacidad ante la videovigilancia. La oposición es además activa, pues desarrolla acciones de boicot y muestras públicas de rechazo. El conformismo generalizado se mantiene, pero puede notarse el incremento de la crítica hacia la vigilancia, reflejado en un cambio de tendencia entre 2008 y 2011. El paso hacia la revisión crítica 
que se inició hace lustros a nivel académico, y de forma más reciente en el ámbito mediático, puede que comience a encontrar eco en el conjunto de la sociedad.

Considerados todos los elementos planteados, el porqué de la videovigilancia parece no residir tanto en las necesidades de una sociedad asustada, como en la ventana de oportunidad que abren las urgencias de un marco institucional deslegitimado y una sociedad que busca culpables. Todas las hipótesis planteadas parecen indicar que la aparente sordera de responsables políticos y operadores ante la ineficacia de la videovigilancia esconde una constelación de modificaciones y transformaciones en la percepción pública de la inseguridad, en la gobernanza urbana y en la conceptualización del riesgo. El círculo de la redundancia e inutilidad de la eficiencia y la eficacia, pues, se cierra. No importa si reduce la delincuencia o mejora la sensación de seguridad: la videovigilancia parece imponerse porque es una solución inmediata, fácil de implementar, susceptible de levantar poca oposición y que "funciona", por tanto, incluso cuando es inútil como herramienta de gestión de la inseguridad.

\section{Referencias bibliográficas}

Agencia Española de Protección de Datos (AEPD). (2009). Memoria 2009. Madrid: Autor. En http://goo.gl/Q9Kg28

Armitage, R. (2002). To cctv or not to cctv?: A review of current research into the effectiveness of cctv systems in reducing crime. Nacro Briefing Note.

Bannister, J. \& Kearns, A. (2009). Tolerance, respect and civility amid changing cities. En A. Millie (Ed.), Securing respect: Behavioural expectations and antisocial behaviour in the uk (pp. 171-192). Bristol: Policy Press.

Bauman, Z. (2000). Liquid modernity. Cambridge: Polity Press.

Bétin, C., Martinais, E. \& Renard, M. C. (2003). Sécurité, vidéosurveillance et construction de la déviance: l'exemple du centre-ville de Lyon. Déviance et Société, 27(1), 3-24. doi: $10.3917 / \mathrm{ds} .271 .0003$

Blanco, I. (2004). Governance urbana i polítiques d'inclusió sòcio-espacial. Tesis doctoral, Universitat Autònoma de Barcelona.

Brenner, N. \& Theodore, N. (2002). Spaces of neoliberalism: Urban restructuring in Western Europe and North America. Oxford: Blackwell.

Bruno, F., Kanashiro, M. \& Firmino, R. (2010). Vigilância e visibilidade. Espaço, tecnologia e identificação. Porto Alegre: Editora Sulina.

Cameron, A., Kolodinski, E., May, H. \& Williams, N. (2008). Measuring the effects of video surveillance on crime in Los Angeles. Los Ángeles, CA: School of Policy, Planning and Development/California Research Bureau. En https://www.library.ca.gov/ crb/08/08-007.pdf

Centro de Investigaciones Sociológicas (cIs), España. (2008). Barómetro Febrero 2008. Madrid: Autor. En http://www.cis.es/cis/export/sites/default/-Archivos/Marginales/2740 _2759/2754/e275400.html 
Centro de Investigaciones Sociológicas (CIS), España. (2009). Barómetro Septiembre 2009. Madrid: Autor. En http://www.cis.es/cis/export/sites/default/-Archivos/ Marginales/2800_2819/2812/es2812.pdf

Centro de Investigaciones Sociológicas (CIS), España. (2011). Barómetro Mayo 2011. Madrid: Autor. En http://www.cis.es/cis/export/sites/default/-Archivos/ Marginales/2880_2899/2888/Es2888.pdf

Cerezo Domínguez, A. \& Díez Ripollés, J. L. (2010). La videovigilancia en las zonas públicas: su eficacia en la reducción de la delincuencia. Boletín Criminológico, 121 (junio-julio), 1-4. En http://dialnet.unirioja.es/servlet/articulo? codigo=3712406

Cochrane, A. (2007). Understanding Urban Policy: A critical approach. Oxford: Blackwell Publishing.

Cohen, M., March, J. \& Olsen, J. (1972). A garbage can model of organizational choice. Administrative Science Quarterly, 17(1), 1-25. En http://goo.gl/tSkWZf

Coleman, R. (2004). Reclaiming the streets: Surveillance, social control and the City. Devon: Willan Publishing.

Cook, I. R. (2008). Mobilising urban policies: The policy transfer of us business improvement districts to England and Wales. Urban Studies, 45(4), 773-795. doi: $10.1177 / 0042098007088468$

Crawford, A. (1999). The local governance of crime: Appeals to community and partnerships. Oxford: Oxford University Press.

Deisman, W. (2003). cctv: Literature review and bibliography. Report of the Research and Evaluation Branch of the Community, Contract and Aboriginal Policing Services Directorate. Ottawa: Royal Canadian Mounted Police. En http://www.academia. edu/357626/cCTv_Literature_Review_and_Bibliography

Díez Ripollés, J. L. \& Cerezo Domínguez, A. I. (2009). La prevención de la delincuencia callejera mediante videocámaras: Regulación jurídica y eficacia. Política criminal, 4(7), 171-196. En http://www.scielo.cl/pdf/politcrim/v4n7/art06.pdf

Ditton, J. (2002). Crime and the city: Public attitudes to CCTv in Glasgow. British Journal of Criminology, 40, 692-709.

Dixon, D. \& Maher, L. (2005). Policing, crime and public health. Lessons for Australia from the "New York Miracle". Criminal Justice, 5(2), 115-143. http://dx.doi. org/10.1177/1466802505053494

Dixon, J. Levine, M. y McAuley, R. (2003). Street drinking legislation, cctv and public space: exploring attitudes towards public order measures. Londres: Home Office.

Dolowitz, D. \& Marsh, D. (1996). Who learns what from whom: A review of the policy transfer literature. Political Studies, 44, 343-357. doi: 10.1111/j.1467-9248.1996. tb00334.x

Douillet, A. C., Dumoulin, L. \& Germain, S. (2009). Un dispositif socio-technique à la loupe: genèse et banalisation de la vidéosurveillance dans trois villes françaises. Congrés de la Société Québecoise de la Science Politique, Ottawa. En http://www.researchgate.net/ profile/Laurence_Dumoulin

Foucault, M. (1975/1995). Surveiller et punir. Naissance de la prison. París: Gallimard.

Fox Gotham, K. (2002). Marketing Mardi Gras: Commodification, spectacle, and the political economy of tourism in New Orleans. Urban Studies, 39(1), 1735-1756. doi: 10.1080/0042098022000002939 
Fussey, P. (2004). New Labour and New Surveillance: Theoretical and political ramifications of CCTV Implementation in the UK. Surveillance \& Society, 2(2/3), 251-269. En http:// www.surveillance-and-society.org/articles $2 \% 282 \% 29 /$ newlabour.pdf

Fyfe, N. R. (1998). Images of the street: Planning, identity, and control in public space. Londres: Routledge.

Fyfe, N. R. \& Bannister, J. (1996). City watching: Closed circuit television surveillance in public spaces. Area, 28(1), 37-46. En http://www.jstor.org/stable/20003625

Galdon-Clavell, G. (2011). Local surveillance in a global world: Zooming in on the proliferation of CCTV in Catalonia. Information Polity, 16(4), 319-338. doi: 10.3233/IP-2011-0251

Galdon-Clavell, G. (2012): Per què la videovigilància? Seguretat, tecnologia i politiques urbanes. Tesis Doctoral no publicada. Institut de Govern i Polítiques Públiques, Universitat Autònoma de Barcelona.

Galdon-Clavell, G. \& Pybus, M. (2011). Crisis económica y gestión de la inseguridad ciudadana: los mapas de delincuencia. Revista Catalana de Seguretat Pública, 24, 79105. En http://www.raco.cat/index.php/rcsp/article/viewFile/244856/327920

Galdon-Clavell, G., Zuloaga, L. \& Romero, A. (2012). сстv in Spain: an empirical account of the deployment of video-surveillance in a Southern-European country. Information Polity, 17(1), 57-68. doi 10.3233/IP-2011-0254

Garland, D. (2001). The culture of control: Crime and social order in contemporary society. Oxford: Oxford University Press.

Gill, M., Bryan, J. \& Allen, J. (2007). Public Perceptions of cCTV in Residential Areas: "It Is Not As Good As We Thought It Would Be". International Criminal Justice Review, 17(4), 304-324.

Gill, M. \& Spriggs, A. (2005). Assessing the impact of cctv: Home Office Research Study 292. Home Office Research, Development and Statistics Directorate, 44. Londres: Home Office.

Goetz, E. G. \& Clarke, S. E. (1993). The new localism: Comparative urban politics in a global era. Newburg Park, CA: Sage.

Greenberg, M. (2008). Branding New York. How a city in crisis was sold to the world. Nueva York: Routledge.

Groombridge, N. (2008). Stars of cCTv? How the Home Office wasted millions - a radical 'Treasury/Audit Commission' view. Surveillance \& Society, 5(1), 73-80. En w.surveillance-and-society.org/articles $5 \% 281 \% 29 /$ stars.pdf

Groombridge, N. \& Murji, K. (1994). As easy as AB and cctv. Policing, 10(4), 283-290. [Versión digitalizada en http://publiccriminology.weebly.com/lsquoas-easy-as-ab-andcctvrsquo.html].

Heilmann, E. (2003). La vidéosurveillance, une réponse efficace à la criminalité? Criminologie, 36(1), 89-102. doi: 10.7202/006554ar

Hempel, L. \& Töpfer, E. (2004). сcтv in Europe. Urban eye Final Report. Berlín: Centre for Technology and Society. En http://goo.gl/1XPQnk

Hempel, L. \& Töpfer, E. (2009). The Surveillance Consensus. European Journal of Criminology, 6(2), 157-177. doi: 10.1177/1477370808100544

Hier, S. P. (2010). Panoptic dreams. Streetscape video surveillance in Canada. Londres: Routledge. Jacobs, J. (1992). Death and life of great American cities. Nueva York: Vintage Books. (Original publicado en 1961). 
John, P. (1998). Analysing Public Policy. Londres: Continuum.

Jones, G. W. \& Stewart, J. D. (1985). The case for local Government. Londres: Allen \& Unwin.

Kanashiro, M. M. (2008). Surveillance cameras in Brazil: exclusion, mobility regulation and the new meanings of security. Surveillance \& Society, 5(3), 270-289. En http://goo.gl/ CAZCbU

Kelling, G. L. \& Coles, K. M. (1996). Fixing broken windows: Restoring order and reducing crime in our communities. Nueva York: Touchstone.

Klauser, F. (2009). Lost surveillance studies: A critical review of French work on cCTv. Surveillance \& Society, 6(1), 23-31. En http://goo.gl/Gdg9pj

Koskela, H. (2003). Cam Era - the contemporary urban Panopticon. Surveillance\& Society, 1(3), 292-313. En http://www.surveillance-and-society.org/articles1\%283\%29/camera.pdf

Le Galès, P. (1999). Is political economy still relevant to study the culturalization of cities? European Urban and Regional Studies, 6(4), 295-302. doi: 10.1177/096977649900600402

Lefebvre, H. (1996). Writings on Cities. Cambridge: Blackwell.

Leman-Langlois, S. (2009). Public perceptions of camera surveillance. En D. Lyon, A Report on Camera Surveillance in Canada. Part One (pp. 41-52). Ottawa: scan. En http:// goo.gl/RnNywe

Levan, V. (2009). Mesurer les effets de la sécurisation des quartiers populaires : un état des lieux de la littérature anglo-américaine. Déviance \& Société, 33(1), 95-113. doi: $10.3917 /$ ds.331.0095

Lyon, D. (2009). Introduction. A report on camera surveillance in Canada. Part One. Ottawa: SCAN.

Mazerolle, L., Hurley, D. \& Chamlin, M. (2002). Social behavior in public space: An analysis of behavioral adaptations to CCTV. Security Journal, 15(3), 59-75. doi:10.1057/ palgrave.sj. 8340118

McCann, E. (2010). Urban policy mobilities and global circuits of knowledge: Toward a research agenda. Annals of the Association of American Geographers, 101(1), 107-130. doi: 10.1080/00045608.2010.520219

Miller, J. (2006). Methodology for an evaluation of CCTV in Malaga. Paper presentado al III Congreso de la Sociedad Española de Criminología. Madrid, 5-7 abril.

Millie, A. (2009). Anti-social behaviour. Maidenhead, Berkshire: Open University Press.

Mitchell, K. \& Beckett, K. (2008). Securing the global city: Crime, consulting, risk, and ratings in the production of urban space. Indiana Journal of Global Legal Studies, 15(1), 75100. En http://www.repository.law.indiana.edu/ijgls/vol15/iss1/5/

Murakami Wood, D. (2009). The surveillance society. Questions of history, place and culture. European Journal of Criminology, 6(2), 179-194. doi: 10.1177/1477370808100545

Murakami Wood, D., Ball, K., Lyon, D., Norris, C. \& Raab, C. (2006). A Report on the Surveillance Society. For the Information Commissioner by the Surveillance Studies Network. Full report. En http://news.bbc.co.uk/2/shared/bsp/hi/pdfs/02_11_06_ surveillance.pdf

Nincic, M. \& Cusak, T. R. (1979). The political economy of us military spending. Journal of Peace research, 16(2), 101-115. En http://goo.gl/7XGgTU

Norris, C. (2003). From personal to digital. ссту, the Panopticon, and the technological mediation of suspicion and social control. En D. Lyon (Ed.), Surveillance as social sorting: privacy, risk, and digital discrimination (pp. 249-281). Londres: Routledge. 
Peck, J. (2002). Political economies of scale: Fast policy, interscalar relations and neoliberal workfare. Economic Geography, 78(3), 331-360. doi: 10.1111/j.1944-8287.2002. tb00190.x

Peck, J. \& Theodore, N. (2001). Exporting workfare/importing welfare-to-work:exploring the politics of Third Way policy transfer. Political Geography, 20(4), 427-460. http:// dx.doi.org/10.1016/S0962-6298(00)00069-X

Phillips, C. (1999). A review of cCTv evaluations: Crime reduction effects and attitudes towards its use. Crime Prevention Studies, 10, 123-155. En http://goo.gl/Efv8Ev

Pressman, J. L. \& Wildavsky, A. (1984). Implementation. How great expectations in Washington are dashed in Oakland... Berkeley, CA: University of California Press. (Original publicado en 1973).

Raco, M. (2003). Remaking place and securitising space: Urban regeneration and the strategies, tactics and practices of policing in the UK. Urban Studies, 40(9), 1869-1887. doi: 10.1080/0042098032000106645

Ratcliffe, J. (2006). Video surveillance of public places. Washington: U.S. Department of Justice.

Rochette, B. \& Marchandet, E. (1998). Vidéosurveillance et télésurveillance, médiations techniques et médiations politiques. En M. Ansidiei, D. Dubois, D. Felury \& B. Munier (Eds.), Les risques urbains. Acteurs, systèmes de prevention (pp. 185-206). París: Anthropos.

Rodríguez Borges, R. \& Camps, V. (2010). El discurso del miedo. Madrid: Plaza y Valdés.

Samatas, M. (2004). Surveillance in Greece. From anti-communist to consumer surveillance. Nueva York: Pella.

Sassen, S. (1991). The global city. Princeton: Princeton University Press.

Sassen, S. (2006). Cities in a world economy. Newbury Park, Ca: Pine Forge Press. (Original publicado en 1994).

sCan/The Surveillance Studies Centre. (2009). A Report on Camera Surveillance in Canada. Part one. Ottawa: SCAN.

Short, E. \& Ditton, J. (1995). Does CCTV affect crime? cctv Today, 2(2), 10-12.

Solesbury, W. (1993). Reframing urban policy. Policy and Politics, 2(1), 31-38. http://dx.doi. org/10.1332/030557393782453989

Stone, D. (1999). Learning lessons and transferring policy across time, space and disciplines. Politics, 19(1), 51-59. doi: 10.1111/1467-9256.00086

Stone, D. (2000). Learning lessons, policy transfer and the international diffusion of policy ideas. CSGR Working Paper 69/01. Centre for the Study of Globalisation and Regionalisation, University of Warwick. En http://wrap.warwick.ac.uk/2056/1/ WRAP_Stone_wp6901.pdf

Sutton, A. \& Wilson, D. (2002). Open-street CCTV in Australia: The politics of resistance and expansion. Surveillance \& Society, 2(2/3), 310-322. En http://goo.gl/mL7iRU

Tilley, N. (1993). Understanding car parks, crime and cctv: Evaluation lessons from safer cities. Crime Prevention Unit Paper 42. Londres: Home Office. En http://goo.gl/SBGVk1

Von Hirsch, A. (2000). The ethics of public television surveillance. En D. Von Hirsch, D. Garland \& A. Wakefield (Eds.), Ethical and Social Perspectives on Situational Crime Prevention (pp. 64-65). Oxford: Hart Publishing.

Webb, B. \& Laycock, G. (1992). Reducing crime on the London underground: An evaluation of three pilot projects. Londres: Home Office. 
Webster, C. W. R. (2009). cCTV policy in the uK: Reconsidering the evidence base. Surveillance and Society, 6(1), 10-22.

Webster, C. W. R., Töpfer, E., Klauser, F. R. \& Raab, C. (2012). Video surveillance. Practices and policies in Europe. Innovation and the Public Sector Series, nº. 18. Ámsterdam: Ios Press.

Welsh, B. C. \& Farringdon, D. P. (2002). Crime prevention effects of closed circuit television: A systematic review. Home Office Research Study no. 252. Londres: Home Office. En http://goo.gl/owFt5y

Welsh, B. C. \& Farringdon, D. P. (2003). Effects of closed-circuit television on crime. Annals of the American Academy of Political and Social Science, 587(1), 110-135. doi: $10.1177 / 0002716202250802$

Wilson, J. Q. \& Kelling, G. L. (1982). Broken windows: The police and neighborhood safety. Atlantic Monthly, 249, 29-38. En http://www.theatlantic.com/magazine/ archive/1982/03/broken-windows/304465/

Zukin, S. (1995). The cultures of cities. Cambridge: Wiley-Blackwell.

Zuloaga, Aitor L. \& Romero, A. (2012). сстv in Spain: an empirical account of the deployment of video-surveillance in a Southern-European country. Information Polity, 17(1). doi 10.3233/IP-2011-0254 
